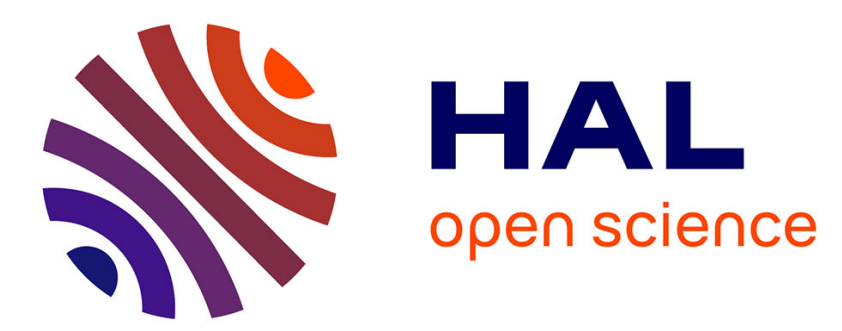

\title{
A MODEL FOR THE CALCULATION OF SURFACE ENERGY AND SURFACE TENSION OF LIQUID METALS
}

\author{
S. Amokrane, Jean Pierre Badiali, M. Rosinberg, J. Goodisman
}

\section{- To cite this version:}

S. Amokrane, Jean Pierre Badiali, M. Rosinberg, J. Goodisman. A MODEL FOR THE CALCULATION OF SURFACE ENERGY AND SURFACE TENSION OF LIQUID METALS. Journal de Physique Colloques, 1980, 41 (C8), pp.C8-783-C8-786. 10.1051/jphyscol:19808194 • jpa-00220298

\section{HAL Id: jpa-00220298 https://hal.science/jpa-00220298}

Submitted on 1 Jan 1980

HAL is a multi-disciplinary open access archive for the deposit and dissemination of scientific research documents, whether they are published or not. The documents may come from teaching and research institutions in France or abroad, or from public or private research centers.
L'archive ouverte pluridisciplinaire HAL, est destinée au dépôt et à la diffusion de documents scientifiques de niveau recherche, publiés ou non, émanant des établissements d'enseignement et de recherche français ou étrangers, des laboratoires publics ou privés. 


\section{A MODEL FOR THE CALCULATION OF SURFACE ENERGY AND SURFACE TENSION OF LIQUID METALS}

S. Amokrane, J.P. Badiali, M.L. Rosinberg and J. Goodisman*

Groupe de Recherche $n^{\circ} 4$ du C.N.R.S., "Physique des Liquides et Electrochimie", Université Pierre

et Marie Curie, 4, place jussieu, 75230 Paris cedex 05, France.

Department of Chemistry, Syracuse University, New York 13210, U.S.A.

Abstract. - Surface energy and surface tension of liquia metals are calculated by applying to Iiquid surface a simple approprlate form of the Lang and Yohn's approach. Resulting values are in reasonable agreement with experiments.

Despite theoretical and technological interest in the study of the surface of liquid metals, few works are devoted to its statistical mechanical description. Lang and Kohn [1] (hereafter referred to as L.K.) have used the Hohenberg-Kohn-Sham theory to calculate the electronic profile $n(z)$ in a semi-infinite positive jellium background. The results they obtain for the surface energy computed on solids are in good agreement with the surface tension of liquids extrapolated to $0^{\circ} \mathrm{K}$. Evans [2] has extended to surface properties the description of the liquid metals in terms of pseudo-atoms. In this model, the electronic profile is given a priori and there are no electrostatic contributions, which are found to be important in L.K. Both of these authors take no account of the transverse collective plasmon modes, this point being a subject of much controversy.

In this paper, an approach to the liquid surface is proposed in the frame of Lang and Kohn's formalism. The liquid nature is taken into account by an ionic density profile $\rho(z)$ of step function type and a simple pair correlation function $g\left(\vec{R}_{p} \vec{R}_{2}\right)$. The electronic density profile is computed by optimization of the zeroth-order surface energy. Computed properties are surface energy $U_{s}$ and surface tension $Y$ for several simple metals.

1. Mode1

For a given configuration $\left\{\vec{R}_{N}\right\}$ of the $N$ ions, and in the adiabatic approximation, the energy of the system can be written as

$$
\mathrm{H}=\mathrm{H}_{\mathrm{i}}+\mathrm{E}\left(\left\{\overrightarrow{\mathrm{R}}_{\mathrm{N}}\right\}\right)
$$

where $H_{i}$ is the ionic hamiltonian and $E\left(\left\{\vec{R}_{N}\right\}\right)$ the ground state energy of the many-electron system in the external potential of the ions. As in L.K., we compute $E\left(\left\{\vec{R}_{N}\right\}\right)$ by considering the difference between the pseudopotentials of the ion cores and the electrostatic potential of the uniform background as a small perturbation. In the jellium, the electron-ion interactions are purely coulombic.

Following the same procedure than Smith [3], we assume that the form of the zeroth-order electron density $n_{0}(z)$ is

$$
\begin{array}{ll}
n_{0}(z)=n\left(1-\frac{e^{\alpha z}}{2}\right) & z \leqslant 0 \\
n_{0}(z)=n \frac{e^{-\alpha z}}{2} & z \geqslant 0.0
\end{array}
$$

where $\mathrm{n}$ is the bulk electron density. The parameter $\alpha$ is obtained by minimization of the functional $E^{\circ}[n]$ corresponding to the reference system (jellium) with conservation of the total number of electrons. $E_{0}[n]$ includes kinetic, exchangecorrelation and electrostatic energies plus an inhomogeneity term which doesn't exist in L.R. . To first order in the perturbation, (1) becomes

$$
\begin{aligned}
& H=H_{i}+E^{0}\left[n_{0}(z)\right]+E^{1}\left[n_{0}(z),\left\{\vec{R}_{N}\right\}\right] \text { with } \\
& E^{1}\left[n_{0}(z),\left\{\vec{R}_{N}\right\}\right]=\int \sum_{i=1}^{N} W\left(\vec{R}_{i}-\vec{r}\right) n_{0}(z) d \vec{r}+ \\
& z \int \frac{D_{0}(z) n_{0}\left(z^{\prime}\right)}{\left|\vec{r}-\vec{r}^{\prime}\right|} d \vec{r} d \vec{r},
\end{aligned}
$$

where $W$ is the electron-ion pseudopotential, $z$ the valence of the ion and $\rho_{0}(z)$ the jellium density profile (in this paper all formulas are given in atomic units). $H_{i}$ includes the kinetic energy of the ions, the1rcoulomb energy and a Born-Mayer type potential which represents the core repulsion.

In order to find the internal energy of the system, one has to take the statistical average of (3) over the configurations $\left\{\vec{R}_{N}\right\}$. With no adsorption on the Gibbs dividing plane, the surface energy $U_{s}$ can be obtained from experiments by the thermodynamic relation $U_{S}=Y-T \frac{\partial Y}{\partial T}$.

$\mathrm{U}_{\mathrm{s}}$ can be written as the sum of four contributions $U_{S}=U_{0}+U_{p s}+U_{c}+U_{B, M}$ 
- $\mathrm{U}_{\mathrm{o}}$ is the surface energy of the reference system, obtained from $\mathrm{E}^{\circ}\left[\mathrm{n}_{\mathrm{o}}(z)\right]$ and the self electrostatic energy of the jellium, by substracting the bulk contributions. When using PinesNozieres formula for exchange-correlation terms we have

$$
\begin{aligned}
& \mathrm{U}_{\circ}=-1.6423 \frac{\mathrm{n}^{5 / 3}}{\alpha}+0.25037 \frac{\mathrm{n}^{4 / 3}}{\alpha}+ \\
& 0.006584 \frac{\mathrm{n}}{\alpha}+\mathrm{n} \alpha \frac{\log 2}{72}+\frac{\pi \mathrm{n}^{2}}{2 \alpha^{3}}
\end{aligned}
$$

The values obtained for $\alpha$ by minimization are very close to Smith's ones.

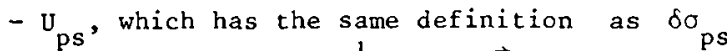
in L.K., is associated to $E^{1}\left[n_{0}(z),\left\{\vec{R}_{N}\right\}\right]$ and depends on the ionic density profile $\rho(z)$. As in Fowler's model we assume for $\rho(z)$ a step function. If we adopt the local Aschroft pseudopotential, we have for $\mathrm{U}_{\mathrm{ps}} \frac{\dot{2}_{\mathrm{nn}}{ }^{2}}{\mathrm{U}^{3}}\left(1-\alpha \mathrm{R}_{\mathrm{c}}-\mathrm{e}^{-\alpha R_{\mathrm{c}}}\right)$ where $R_{c}$ is the cutoff radius.

- U, which can be compared to the classical cleavage energy of solids, represents the ionic part of the surface coulomb energy minus the ionic part of the surface energy of the jellium, already in $\mathrm{U}_{0}$

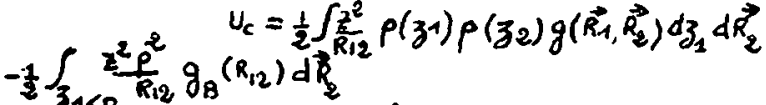

$$
\begin{aligned}
& -\frac{1}{2} \int_{\frac{1}{R_{1}}}^{2}\left[p_{6}\left(z_{2}\right) p_{2}\left(z_{2}\right)-\rho_{2}^{2}\left(z_{1}\right)\right] d z_{1} d k_{2} \\
& \text { where } g_{B}\left(K_{12}\right) \text { is the radial distrioution function }
\end{aligned}
$$

(r.d.f.) of the bulk liquid and $\rho$ the bulk ionic density.

To go further, some appropriate approximations for $g\left(\vec{R}_{1}, \vec{R}_{2}\right)$ still have to be made. If we take $g\left(\vec{R}_{1}, \vec{R}_{2}\right)=g_{B}\left(R_{12}\right)$ as in Fowler's model, we violate the electroneutrality condition that the charge, from ions and electrons, surrounding an fon at $\vec{R}_{1}$ should be $-\mathrm{Z}$ :

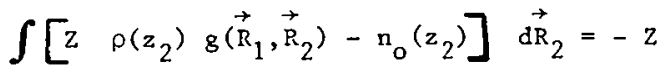

Violation of the condition can have drastic consequences in coulombic systems as shown by one of us (J.G.) [4]; we incorporate electroneutrality into $g\left(\vec{R}_{1}, \vec{R}_{2}\right)$ by writing

$$
g\left(\vec{R}_{1}, \vec{R}_{2}\right)-1=\left[g_{B}\left(R_{12}\right)-1\right] f\left(z_{1}\right) f\left(z_{2}\right)
$$

Substituting into (9) gives an integral equation which may be solved iteratively starting with $f=f^{(0)}=1$. The first iteration yields $f^{(1)}\left(z_{1}\right)=\left[\int \rho\left(z_{2}\right)\left[g_{B}\left(R_{12}\right)-1\right] d \vec{R}_{2}\right]-1$ and replacing $f\left(z_{1}\right) \quad f\left(z_{2}\right)$ by $f^{(1)}\left(z_{1}\right) f^{(0)}\left(z_{2}\right)$ gives

results close to those from a more accurate choice [9].

In the bulk fluid, correlations for small $r$ are well described by an "effective" hard-sphere potential. Here we use for the r.d.f. $g_{B}(r)$ of the bulk phase a simplified form

$g_{B}(r)=0 \quad r<\sigma ; g_{B}(r)=g(r) \sigma \leqslant r \leqslant \delta$

$g_{B}(r)=1 \quad r>\delta$

where $g(\sigma)$ is given by the Percus-Yevick equation and $\delta$ is calculated from the relation (see eq. 9) : $\int[\vec{g}(r)-1] d \vec{p}=-\rho^{-1}$

With all these assumptions, we can write after numerical integration $\mathrm{U}_{c}=\mathrm{n} \mathrm{Z}$ a $(n)$

This form is similar to Lang and Kohn's cleavage energy, $a(\eta)$ which depends only on the packing fraction $\eta=\frac{\pi}{6} \rho \sigma^{3}$ is given in Table 1 for different values of $n$

$(n: a(n): b(\eta))$

$(0.3: 0.010: 0.036)$

$(0.35: 0.036: 0.037)$

$(0.40: 0.068: 0.039)$

$(0.45: 0.099: 0.040)$

$(0.50: 0.131: 0.041)$

$(\quad: \quad: \quad 1)$

- $\mathrm{U}_{\mathrm{B} . \mathrm{M}}$ the last contribution to $\mathrm{U}_{\mathrm{s}}$, comes from Born-Mayer energy. We have found that this contribution is negligible because its range (about $2 \mathrm{R}_{c}$ ) is generally much smaller than $\sigma$, when we take for $\eta$ values appropriate to liquid state.

Starting from (3) it is possible to define the free energy of the system and calculate its surface tension $\gamma$ by differentiation. Using the standard procedures and arranging the different terms in $Y$ as we did in $U_{s}$, we get after some algebra

$$
Y=U_{o}+U_{p s}+\gamma_{c}+\gamma_{B \cdot M}
$$

with $\gamma_{c}=n$ z b (n)

The variation of $b(n)$ with $n \dot{i} \dot{s}$ given in Table 1 (detailed calculation for $a(n)$ and $b(n)$ will be given elsewhere [6]). For the same reason as before, the Born-Mayer term $\gamma_{B, M}$ is negligible.

2. Results for $U_{s}$ and $Y$

Our results for $U_{s}$ and $\gamma$ are given in Table II for several metals and compared to experiments. The different contributions are also given. The values of $n$ and $R_{c}$ are the same as in L.K. for easier comparison. Experimental values are taken from L.E. Murr [5]. 
$\left(\quad: \mathrm{U}_{\mathrm{o}}: \mathrm{U}_{\mathrm{ps}}: \mathrm{U}_{\mathrm{c}}: \mathrm{U}_{\mathrm{s}}: \mathrm{U}_{\mathrm{S}}^{\text {exp }}: \gamma_{\mathrm{c}}: \gamma: \gamma^{\exp }\right)$

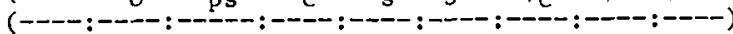
(Cs : 51: -21: 90:120: 88: 81:111:60) ( $\mathrm{Rb}: 62:-29: 112: 145: 104: 101: 134: 76$ ) $(\mathrm{K}: 70:-30: 131: 171: 138: 118: 158: 101)$ ( $\mathrm{Na}: 115:-84: 254: 285: 228: 228: 259: 191$ ) ( $\mathrm{Li}: 143:-143: 467: 467: 461: 420: 420: 398$ ) ( $\mathrm{Hg}: 51:-775: 1726: 1002: 835: 1553: 829: 540$ ) ( $\left.\mathrm{Zn}_{\mathrm{n}}:-251:-1560: 2630: 819: 888: 2367: 556: 770\right)$ ( A1 : $-795:-2368: 5442: 2279: 1302: 4897: 1734: 866$ )

Table II - Different contributions to $U_{s}$ and $\gamma$ for simple metals. Units are dyne $/ \mathrm{cm}$.

Values of $U_{0}$ are similar to Lang and Kohn's results, although the partition between the various contributions may be quite different (in L. K. there is no gradient expansion term which gives here, for instance, 26 dyne $/ \mathrm{cm}$ for $\mathrm{Cs}$ and 500 dyne/cm for $\mathrm{Al}$ ).

The pseudopotential contribution $U_{p s}$ is negative, in opposition to L.K. in the case of the lattice model. $U_{p s}$ is rather sensitive to the choice of $R_{c}$, especially at high density : for $A 1$, a modification of $10 \%$ in $R_{c}$ changes $U_{s}$ an $\gamma$ by 350 dyne $/ \mathrm{cm}$. The presence of oscillations in the electronic profile may substantially modify the result for $U_{p s}[1]$. To test the order of magnitude of this effect, we have used in $U_{p s}$ another form of $n_{0}(z)$ :

$$
\begin{array}{ll}
n_{0}(z)=n\left(1-\mu e^{\alpha z} \cos 2 k_{F} z\right) & z \leqslant 0 \\
n_{0}(z)=n v e^{-\beta z} & z>0
\end{array}
$$

This form roughly simulates Friedel oscillations, although they have no exponential decay. Unknown parameters are obtained by continuity conditions of $n_{0}(z)$ and $d n_{0}(z) / d z$ at $z=0$, and overall electroneutrality. Oscillations in $n_{0}(z)$ give an increase of 77 dyne/cm in $U_{p s}$ for $L i, 15$ for $C s$ and 1800 for $A l$. For all metals, $U_{p s}$ remains negative, unlike the corresponding term in L.K.

- The contribution $U_{c}$ is very sensitive to the value of the packing fraction $\eta$, as shown in table 1 , through the variation of $a(n)$. Bulk thermodynamic properties of liquid metals are known to be well described by putting $n \approx 0.45$ in the hard sphere model. With this value, $\mathrm{U}_{\mathrm{c}}$ becomes the main contribution to the surface energy. For $\mathrm{A} 1, \mathrm{U}_{\mathrm{C}}=12447$ dyne $/ \mathrm{cm}$ when $U_{s}=9284 \mathrm{dyne} / \mathrm{cm}$, and for Cs $U_{c}=205$ dyne $/ \mathrm{cm}$, when $U_{s}=235$ dyne $/ \mathrm{cm}$. This contribution is much greater than the cleavage energies of Lang and Kohn, corresponding to the [111] plane for f.c.c. lattice and [110] plane for b.c.c. lattice. It is comparable to cleavage energies corresponding to less densely packed faces.

- For a liquid metal it is in fact difficult to consider that the ions near the surface are correlated as in the bulk where they are totally screened by the electrons.

If the description of correlations in terms of a hard sphere model remains valid near the surface, we can expect a value of $\sigma$ somewhat smaller than its value in the bulk, and then effective values of $n$ smaller than 0.45 . It is clear that an effective value of $n$ takes into account other effects, such as a modification of the step density profile as suggested in [7], or higher order contributions to the energy of the system. On the other hand, we note that $\gamma_{c}$ is less sensitive to the variation of $n$ than $\mathrm{U}_{c}$. Rather than fitting $n$ for each metal as done in [7], an average value of $\eta=0.36$ has been taken in table 2 . With this value, the magnitude of calculated results for $U_{S}$ and $\gamma$ are in reasonable agreement with experimental data. We emphasize that our model permits us to compute simultaneously $\mathrm{U}_{s}$ and $\gamma$ and it is satisfactory that with the same ingredients, their magnitudes are at least reasonable and close one to the other. This is not the case with other treatments nevertheless the remaining difference cannot be attributed to the influence of the temperature (entropy term), because in this approach $U_{S}$ and $\gamma$ depend on $T$ only through the bulk densities which are rather independent of $T$. A more realistic treatment of the ionic profile should consider its dependence with temperature, in order to satisfy the thermodynamic relation

$$
u_{s}=\gamma-T \frac{\partial \gamma}{\partial T}
$$

In our calculations, it is clear that changes in the two profiles may induce notable modifications in the different contributions to $U_{s}$ and $\gamma$. A self consistent determination of the two profiles is in progress [8].

Our calculations also show the importance of respecting the electroneutrality condition when approximating the anisotropic pair distribution $g\left(\vec{R}_{1}, \vec{R}_{2}\right)$.

We finally note that our model gives rise to a dipole barrier at the surface, in contrast to the work of Evans [2]. The values of the dipole barrier are the same as those computed by Smith [3]. 
All these results show that this simple model may be considered as a usefull step in the understanding of thermodynamic surface properties of liquid metals.

\section{REFERENCES}

[1] LANG, N.D., KOHN, W.,Phys.Rev.B, 1, 4555 (1970).

[2] EVANS, R., J. Phys. C, 7, 2808 (1974).

[3] SMITH, J.R., Phys. Rev., 181, 522 (1969).

[4] PASTOR, R.W., GOODISMAN J., J. Chem. Phys., $\underline{68}$, 3654 (1978).
[5] MURR, L.E., Interfacial Phenomena in Metals and alloys. Addison Wesley Pred. Com. London 1978.

[6] AMOKRANE, S., BADIALI, J.P., ROSINBERG, M.I., GOODISMAN, J. (to be published).

[7] ITAMI, T., SHIMOJI, M., J. Phys. F., 9, L15 (1979).

[8] AMOKRANE S., Thèse 3e cycle.

[9] GOODISMAN, J. , PASTOR, R.W., J. Phys. Chem. 82, 2078 (1978). 